ARTICLE

\title{
Giant anisotropic magnetoresistance in a quantum anomalous Hall insulator
}

\author{
Abhinav Kandala ${ }^{1}$, Anthony Richardella ${ }^{1}$, Susan Kempinger ${ }^{1}$, Chao-Xing Liu $^{1} \&$ Nitin Samarth ${ }^{1}$
}

When a three-dimensional ferromagnetic topological insulator thin film is magnetized out-of-plane, conduction ideally occurs through dissipationless, one-dimensional (1D) chiral states that are characterized by a quantized, zero-field Hall conductance. The recent realization of this phenomenon, the quantum anomalous Hall effect, provides a conceptually new platform for studies of 1D transport, distinct from the traditionally studied quantum Hall effects that arise from Landau level formation. An important question arises in this context: how do these 1D edge states evolve as the magnetization is changed from out-of-plane to in-plane? We examine this question by studying the field-tilt-driven crossover from predominantly edge-state transport to diffusive transport in $\mathrm{Cr}_{x}(\mathrm{Bi}, \mathrm{Sb})_{2-x} \mathrm{Te}_{3}$ thin films. This crossover manifests itself in a giant, electrically tunable anisotropic magnetoresistance that we explain by employing a Landauer-Büttiker formalism. Our methodology provides a powerful means of quantifying dissipative effects in temperature and chemical potential regimes far from perfect quantization.

\footnotetext{
${ }^{1}$ Department of Physics, The Pennsylvania State University, University Park, Pennsylvania 16802-6300, USA. Correspondence and requests for materials should be addressed to N.S. (email: nsamarth@psu.edu).
} 
$\mathrm{n}$ the absence of magnetic doping, surface electrons in threedimensional (3D) topological insulators such as $(\mathrm{Bi}, \mathrm{Sb})_{2} \mathrm{Te}_{3}$ are helical Dirac fermions protected from backscattering by time-reversal symmetry ${ }^{1,2}$. When such a $3 \mathrm{D}$ topological insulator is made ferromagnetic by doping with magnetic ions ${ }^{3-9}$ or by integration into magnetic heterostructures ${ }^{10-12}$, time-reversal symmetry is broken. If the magnetization $M$ is perpendicular to the surface, the surface state dispersion is gapped at the Dirac point ${ }^{13,14}$, while $M$ parallel to the surface is expected to keep the Dirac point intact with the origin shifting in momentum-space ${ }^{15}$. In a ferromagnetic topological insulator thin film with out-ofplane magnetic anisotropy ${ }^{16}$, the two surfaces are gapped, albeit with opposite mass. The mass domain wall created along the side walls of films then hosts a massless, one-dimensional (1D), dissipation-free edge state. If the chemical potential is tuned inside the magnetic gap of the surface states, transport is solely restricted to these $1 \mathrm{D}$ edge states, and characterized by a quantized Hall resistivity $\rho_{x y}=h / e^{2}$ (where $h$ and $e$ are the Planck constant and elementary charge, respectively) and (ideally) vanishing longitudinal resistivity $\rho_{x x}=0$. This is known as the quantum anomalous Hall (QAH) state and was recently realized experimentally in thin films of $\mathrm{Cr}_{x}(\mathrm{Bi}, \mathrm{Sb})_{2-x} \mathrm{Te}_{3}$ (refs 6,8,9) at dilution fridge temperatures.

Anisotropic magnetoresistance (AMR) ${ }^{17,18}$ is the response of the longitudinal resistance of a ferromagnet to the angle between the magnetization and direction of current flow. In this work, by tilting the magnetization of QAH insulator in-plane, we observe a novel, giant AMR effect that serves as a powerful quantitative probe of edge transport, even in temperature and chemical potential regimes away from perfect quantization (for instance, at high temperatures compared with the energy scale of the magnetic gap). Tilting the magnetization in-plane closes the magnetic gap and consequently destroys the edge states, leading to a crossover from dissipationless to diffusive transport. We demonstrate that this crossover yields a sharp change in the longitudinal resistivity $\left(\rho_{x x}\right)$ whose angular dependence is unlike classical AMR and show that modelling this dependence can be used to quantify edge-state contributions to transport, and the extent of its inter-mixing with dissipative channels.

\section{Results}

Quantum anomalous Hall effect. The magnetic topological insulator devices used in this study were fabricated from epitaxial films of $\mathrm{Cr}_{x}(\mathrm{Bi}, \mathrm{Sb})_{2-x} \mathrm{Te}_{3}$ grown by molecular beam epitaxy on $\langle 111\rangle \mathrm{SrTiO}_{3}$ (STO) substrates and measured in a vector magnet cryostat down to $280 \mathrm{mK}$ (Fig. 1a, see Methods for details). The large dielectric constant of the STO substrate at cryogenic temperatures allows effective electrical back gating for tuning the position of the chemical potential ${ }^{19}$. Three thin-film devices-A, $\mathrm{B}$ and $\mathrm{C}$, were studied with varying proximity to the QAH state (Table 1). The transport properties of these devices are detailed in Supplementary Figs 1-10 and Supplementary Notes 1-3. Unless otherwise mentioned, the data presented in the main text of this manuscript is from device A-a Hall bar of channel dimensions $1 \mathrm{~mm} \times 0.5 \mathrm{~mm}$ fashioned from a 10 quintuple layer thin film of $\mathrm{Cr}_{0.1}\left(\mathrm{Bi}_{0.5}, \mathrm{Sb}_{0.5}\right)_{1.9} \mathrm{Te}_{3}$. The ferromagnetic Curie temperature of this film is $T_{\mathrm{C}} \sim 8 \mathrm{~K}$, obtained from the temperature dependence of the transverse resistivity $\rho_{x y}$. As shown in Fig. 1b,c, even at $280 \mathrm{mK}$, the AHE in device A takes a maximum value $\rho_{x y} \sim 0.95 h / e^{2}$ $\left(\sigma_{x y} \sim 0.99 h / e^{2}\right)$ and the longitudinal resistivity its minimum value $\rho_{x x} \sim 0.25 h / e^{2}$, both at zero field, when the gate voltage is tuned to its value at charge neutrality $V_{\mathrm{G}}{ }^{0}$. This non-vanishing $\rho_{x x}$, accompanied by $\rho_{x y}$ taking near-quantized values, is indicative of
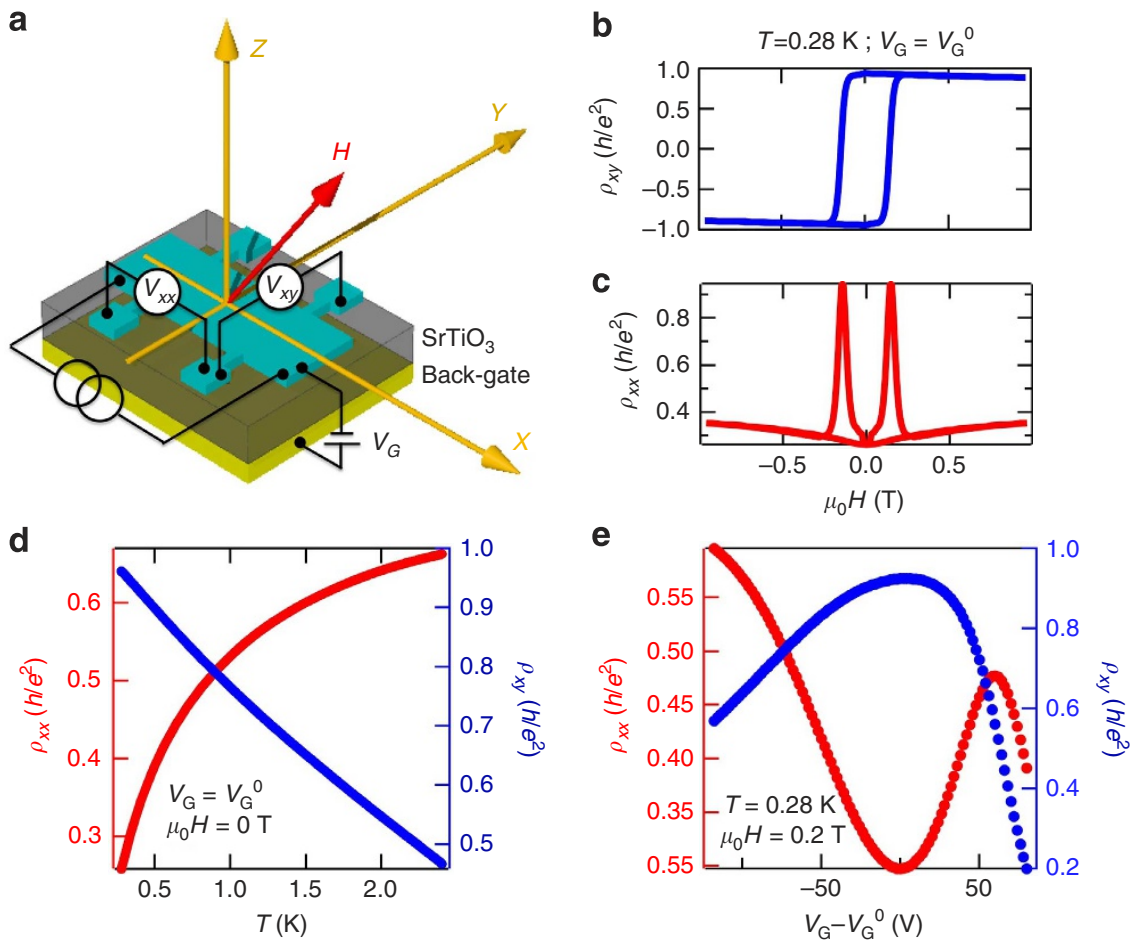

Figure 1 | Quantum anomalous Hall effect (a) Cartoon of device and measurement scheme. Current flows in the $x$ direction, and $z$ is the direction perpendicular to plane. (b) Near-quantized anomalous Hall effect at charge neutrality $\left(V_{\mathrm{G}}=V_{\mathrm{G}}{ }^{0}\right)$ and $T=280 \mathrm{mK}$, with $\rho_{x y}$ taking a maximum value of $0.95 \mathrm{~h} / \mathrm{e}^{2}$ at zero field. (b,c) Corresponding longitudinal magnetoresistance, displaying a minimum $\rho_{x x}$ of $0.25 \mathrm{~h} / \mathrm{e}^{2}$, also at zero field. (d,e) Characteristic signatures of quantum anomalous Hall effect in the temperature and gate voltage dependence, respectively, of $\rho_{x x}$ (red) and $\rho_{x y}$ (blue), after magnetic training, at zero field and $200 \mathrm{mT}$, respectively. As edge-state transport dominates over diffusive transport, the decrease in $\rho_{x x}$ is complemented by a rise in $\rho_{x y}$. 
dissipative channels coexisting with the $1 \mathrm{D}$ chiral edge modes. $\rho_{x x}$ also displays a prominent hysteretic peak at magnetization reversal (Fig. $1 \mathrm{~b}, \mathrm{c}$ ), and takes very large values approaching $260 \%$. This large effect has been interpreted as enhanced backscattering between edge channels in a manner similar to the sharp $\rho_{x x}$ rise in the plateau transition of the quantum Hall effect. Below $3 \mathrm{~K}$, at charge neutrality, $\rho_{x x}$ displays a metallic behaviour as $\rho_{x y}$ approaches quantization (Fig. 1d,e). This metallic behaviour is another signature of edge-state transport and $\rho_{x x}$ recovers its insulating behaviour when the chemical potential is tuned far out of the magnetic gap (Supplementary Fig. 2). Similarly, in the gate voltage dependence, as we tune the chemical potential towards the magnetic gap, $\rho_{x x}$ drops sharply, as $\rho_{x y}$ rises and finally flattens close to its maximum value.

Table 1 | Comparison of transport properties and AMR fitting parameters of three different $\mathrm{Cr}_{x}\left(\mathrm{Bi}_{1} \mathrm{Sb}\right)_{2}{ }_{-x} \mathrm{Te}_{3}$ thin-film devices with varying degrees of dissipation.

\begin{tabular}{cccllc} 
Device & $\boldsymbol{\rho}_{\mathbf{x y}}\left(\boldsymbol{h} / \mathbf{e}^{\mathbf{2}}\right)$ & $\boldsymbol{\rho}_{\mathbf{x x}}(\mathbf{0})\left(\boldsymbol{h} / \mathbf{e}^{\mathbf{2}}\right)$ & \multicolumn{1}{c}{$\boldsymbol{\eta}$} & $\boldsymbol{R}_{\mathbf{d}}^{-\mathbf{1}}$ & AMR (\%) \\
\hline A & 0.95 & 0.25 & 0.66 & 0.62 & 140.9 \\
B & 0.72 & 0.55 & 0.16 & 0.54 & 75.6 \\
C & 0.48 & 2.79 & 0.013 & 0.025 & 100.2
\end{tabular}

AMR, Anisotropic magnetoresistance.

The proximity of devices $A, B$ and $C$ to the ideal $Q A H$ regime is seen by a comparison of their zero-field $\rho_{x x}$ and $\rho_{x y}$ and is indicative of the strength of dissipation. The effect of dissipative channels is quantified by the edge-state transmission coefficient $\eta$, obtained by modelling the out-of-plane AMR of the different devices.
Metal-insulator transition. Having established the signatures of edge-state transport, we now examine their evolution as the magnetization is tilted in-plane by an external $1 \mathrm{~T}$ radial field (Fig. 2). The temperature dependence of $\rho_{x x}$ in Fig. 2a shows a metal-insulator transition as the magnetization is tilted from out-of-plane to in-plane, irrespective of the azimuthal angle (also see Supplementary Fig 5). Similarly, in the gate voltage dependence (Fig. 2b), as the chemical potential is tuned into the magnetic gap, the drop in $\rho_{x x}$ is replaced by a sharp rise when the magnetization is in-plane. This is indicative of a crossover from dominant 1D edge transport to diffusive transport, as the system transitions from a QAH regime to a gapless ferromagnetic topological insulator (when magnetized in-plane). Figure 2a,b already reveals a giant AMR effect when the chemical potential is in the magnetic gap, and this crossover is the origin of the novel angular dependence of the AMR that we now discuss.

Giant anisotropic magnetoresistance. We systematically probe the crossover from edge dominated to diffusive transport as we rotate a $1 \mathrm{~T}$ external magnetic field from perpendicular-to-plane to in-plane. For a $1 \mathrm{~T}$ field, the AMR, defined as $\left(\rho_{x x, \max }-\right.$ $\left.\rho_{x x, \text { min }}\right) / \rho_{x x, \text { min }}$ takes a maximum value $\sim 140 \%$, and its angular dependence is completely inconsistent with the well-known $\cos ^{2} \theta$ angular dependence seen in conventional ferromagnets. To explain these observations, we model the angular dependence using a simplified four-terminal Landauer-Büttiker formalism $I_{i}=\Sigma_{j} G_{i j}\left(V_{i}-V_{j}\right)$, where $I_{i}$ and $V_{i}$ are the current through and the voltage at the $i$ th contact, and $G_{i j}$ is the conductance of the channel between the $i$ th and $j$ th contacts. Our model accounts for the coexistence of both dissipationless edge channels and a

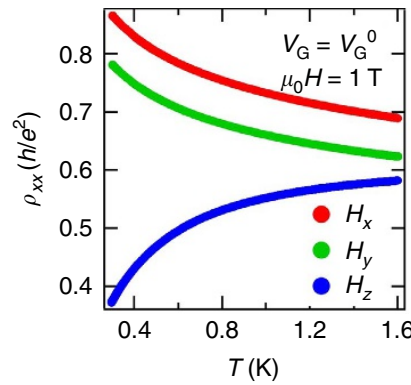

b

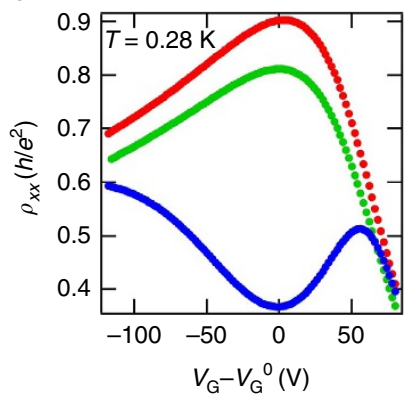

C

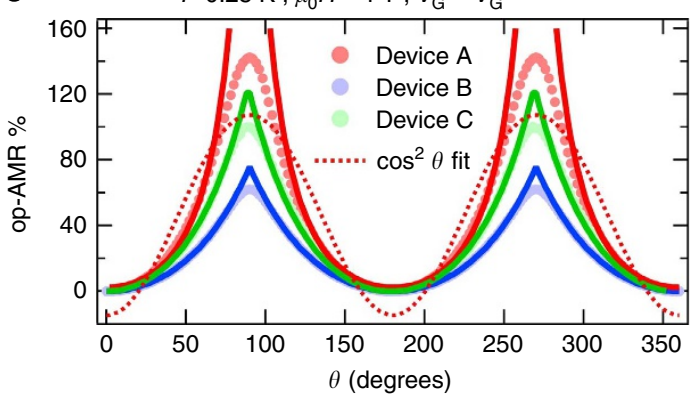

e

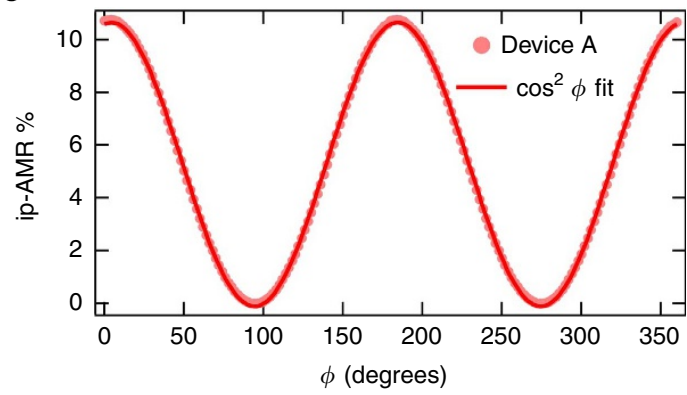

d
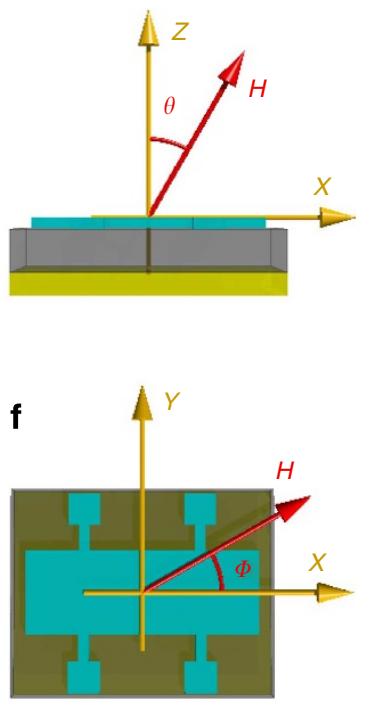

Figure 2 | Magnetization tuned edge-state transport. An external radial field of $1 \mathrm{~T}$ is used for all the data in this figure. (a) Transition from metallic to insulating behaviour in the temperature dependence of $\rho_{x x}$ at $V_{G}=V_{G}{ }^{0}$, as the magnetization is tilted from out-of-plane to in-plane. The red, green and blue curves correspond to the $x, y$ and $z$ directions, respectively. (b) Gate voltage dependence of $\rho_{x x}$ at $T=280 \mathrm{mK}$ when the film is magnetized along the $x$ (red), $y$ (green) and $z$ (blue) directions. (c) Out-of-plane anisotropic magnetoresistance of device A (light red, circles) at charge neutrality, as the field is rotated in the $x-z$ plane. The angular dependence is unexplained by a conventional $\cos ^{2} \theta$ fit (red, dotted line) and is understood by fits (red, bold line) that use an edge-dissipation mixing model. AMR data (circles) and corresponding fits (bold lines) from device B (light blue) and device C (light green), described in the text. (d) Cartoon of the device geometry highlighting field rotation from out-of-plane to in-plane, in the $x-z$ plane. (e) In-plane anisotropic magnetoresistance of device A (light red, circles) at charge neutrality, as the field is rotated in the $x-y$ plane. The angular dependence is explained by a typical $\cos ^{2} \phi$ fit (red, bold line). (f) Cartoon of the device geometry highlighting field rotation in the $x-y$ plane. 
dissipative bulk and surface channels. The dissipative channels likely arise from the gapless, two-dimensional surface Dirac states of the side walls when the chemical potential is in the magnetic gap $^{20}$. We note that these surface states are not gapped because the external magnetic field and the magnetization are always parallel to the plane of the side walls. We cannot exclude other possible origins of dissipation, such as the bulk bands, mid-gap impurity bands, and even the gapped surface states. These details are however not important for the model. The conductance $G_{i j}$ is given by $G_{i j}=T_{i j}\left(e^{2} / h\right)$ where the transmission coefficient $T_{i j}$ takes the form $T_{i j}=\eta \delta_{i, j+1}+t_{i j}$. Here, $\eta$ is the contribution from edge modes that only transmit between leads $j$ and $j+1$, and $t_{i j}$ corresponds to dissipative contributions. From calculations explained in detail in Supplementary Note 4, we derive the following expression for the magnetoresistance:

$$
R=\frac{h}{e^{2}} \frac{1}{\eta\left(2+R_{\mathrm{d}} \eta\right)+R_{\mathrm{d}}^{-1}},
$$

where $R_{\mathrm{d}}$ is a measure of the dissipative resistance (and $R_{\mathrm{d}}^{-1}$ is the dissipative transmission coefficient). Physically, the first term in
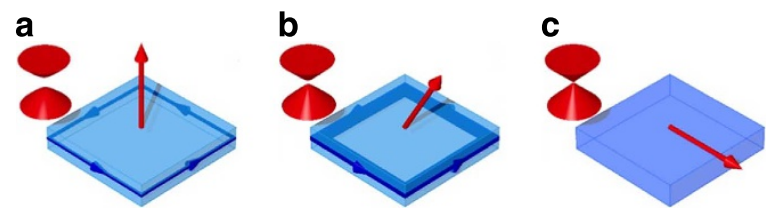

Figure 3 | Edge-dissipation mixing model. A pictorial representation of the physical origins of the giant AMR effect. The penetration length of edge states (depicted in dark blue) is dependent on the size of the magnetic gap $\Delta$, which in turn depends on the perpendicular-to-plane component of magnetization. (a) As the magnetization (red arrows) is tilted in-plane from the intial perpendicular-to-plane configuration, the size of the magnetic gap decreases (illustrated by the energy-momentum dispersions, in red). Consequently, the penetration length of the edge states into the bulk (depicted in light blue) increases (b) eventually leading to complete mixing as the magnetization is tilted perfectly in-plane (c). the denominator is the conductance of an inter-mixed channel, that constitues the edge states scattering into dissipative pathways. The second term though corresponds solely to the dissipative channels. The transmission coefficient for the edge modes $\eta$ depends on the gap $\Delta$ induced by the magnetization. We expect $\eta$ to be suppressed from its maximum value of 1 as the magnetic gap is reduced $\Delta$ : this arises from enhanced intermixing with dissipative channels that accompanies an increase in penetration length $\lambda$ of the edge-state wave functions, where $\lambda=\hbar v_{\mathrm{F}} / \Delta$ where $v_{\mathrm{F}}$ is the Fermi velocity. We thus assume a phenomenological expression for the edge-state transmission as:

$$
\eta=1-e^{-l_{0} / \lambda}=1-e^{-\Delta / \Delta_{0}}
$$

where $l_{0}$ is a length scale that quantifies the strength of the intermixing, and $\Delta_{0}$ is the corresponding energy scale. This expression satisfies the physical requirement at the two extrema: $\Delta \rightarrow$ $0 \Longrightarrow \eta \rightarrow 0$ and $\Delta \rightarrow \infty \Longrightarrow \eta \rightarrow 1$. The angle dependence of the magnetoresistance then arises via the dependence of the magnetic gap on the perpendicular component of field, that is, $\Delta=\Delta_{\mathrm{m}} \cos \theta$. $\Delta_{\mathrm{m}}$ is the size of the magnetic gap, when the film is magnetized out-of-plane. The physics behind the model is depicted in Fig. 3. We now use equation (1) to fit our angular data with two free parameters: $R_{\mathrm{d}}$ and $\Delta^{\prime}$ (defined as $\Delta^{\prime}=\Delta_{0} / \Delta_{\mathrm{m}}$ ). The model clearly fits the data very well, up to tilt angles $\sim 70^{\circ}$, as seen in Fig. $2 \mathrm{~b}$, confirming that the novel, giant AMR effect has its origins in the edge states. The model deviates at large tilt angles when the field approaches the in-plane orientation. In this regime, the dominant resistance is associated with the dissipative channels, whose angular magnetoresistance is not accounted for in our model $\left(R_{\mathrm{d}}\right.$ is treated as a constant).

Beyond explaining the angular dependence of this novel AMR, our model also presents a powerful approach to quantify edgestate contributions to transport in an edge-dissipation mixed QAH insulator. The fitting parameter $\Delta^{\prime}$ can be used to extract the edge transmission coefficient $\eta=1-e^{-1 / \Delta^{\prime}}$. This is used to quantify and compare edge transport contributions in two other samples with varying degrees of dissipation, whose AMR is also
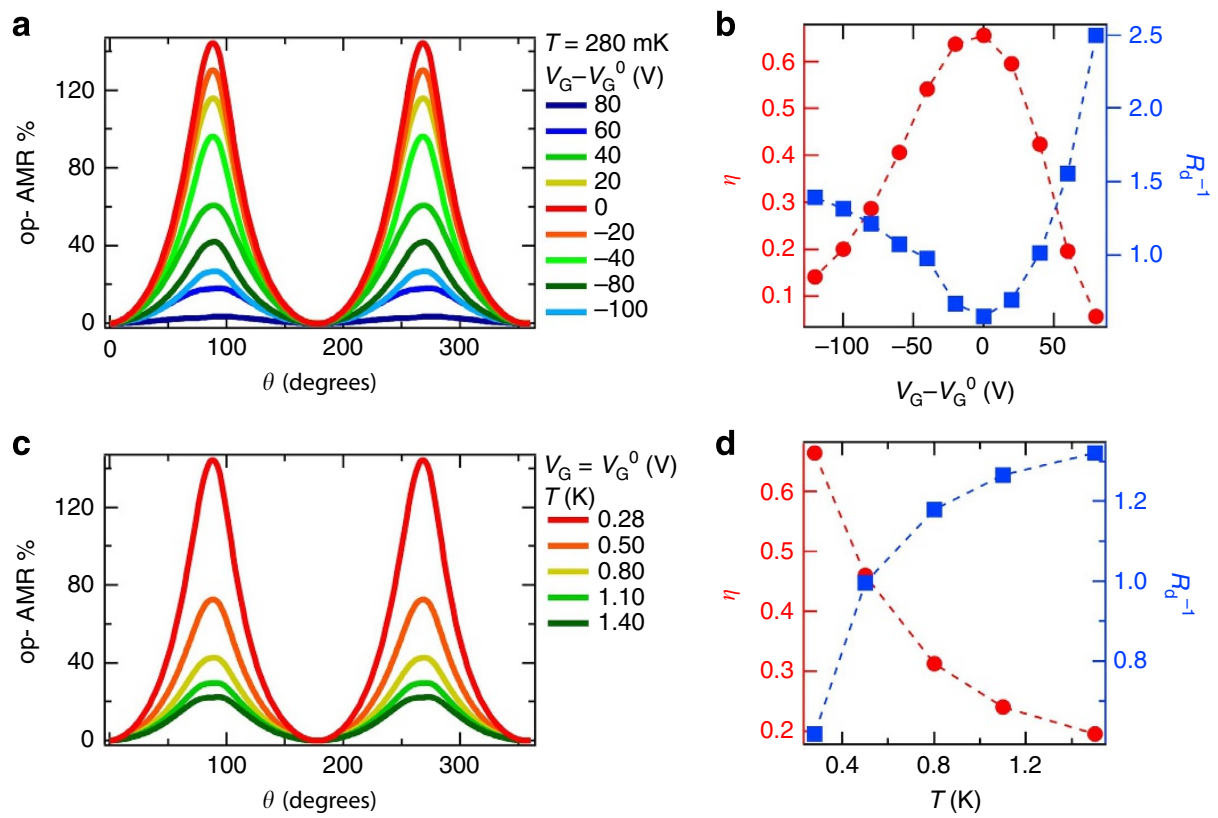

Figure 4 | Giant anisotropic magnetoresistance. (a) Out-of-plane AMR of device A at $T=280 \mathrm{mK}$ for a range of gate voltages. The AMR takes maximum values when the film is tuned to charge neutrality. (b) Gate voltage dependence of the edge-state transmission coefficient $\eta$ (red) and the dissipative transmission coefficient $R_{\mathrm{d}}^{-1}$ (blue) at $280 \mathrm{mK}$, extracted from fits to the data of $\mathbf{a}$. (c) Out-of-plane AMR at $V_{\mathrm{G}}{ }^{0}$ for a range of temperatures.

(d) Temperature dependence of $\eta$ (red) and $R_{\mathrm{d}}^{-1}$ (blue) at $V_{\mathrm{G}}=V_{\mathrm{G}}{ }^{0}$ extracted from fits to the data of $\mathbf{c}$. 
shown in Fig. 2c. The strength of the dissipation is inferred by comparing the zero-field $\rho_{x x}$ and the magnitude of the AHE in these films. The angular dependence and fits to data from devices $\mathrm{B}$ and $\mathrm{C}$, also serves as a test of reproducibility for the giant AMR. Table 1 summarizes the different device characteristics and the extracted fitting parameters, all at charge neutrality and at the same temperature. The extracted values of $\eta$ consistently characterize the degree to which the samples are close to the ideal QAH regime. Also, it is important to point out that the magnitude of the AMR effect consistently depends on the relative values of $\eta$ and $R_{\mathrm{d}}^{-1}$ (equation (1)).

In-plane anisotropic magnetoresistance. As we mentioned earlier, conventional ferromagnets show an AMR described by a $\cos ^{2} \phi$ angular dependence. This behaviour is indeed observed for in-plane measurements (Fig. 2e). Compared the giant AMR, the in-plane AMR only has magnitude of $\sim 10.7 \%$, at charge neutrality (Fig. 2e), and also shows a far weaker temperature dependence (difference between upper two curves in Fig. 2a). With the field and magnetization in-plane, the sample is expected to be in a diffusive regime, since tuning the surface states gapless destroys the edge states. The functional dependence of the in-plane AMR is unchanged with gate voltage (Supplementary Fig. 7).

Gate voltage and temperature dependence of giant AMR. Finally, we present a more detailed picture of dependence of the giant AMR on the gate voltage and temperature. This is shown for device A in Fig. 4. The effect is electrically tunable over an order of magnitude, and is largest in magnitude with the chemical potential in the magnetic gap, when edge-state contributions to transport are maximum. As the chemical potential is tuned out of the magnetic gap, surface state and bulk dissipative channels are populated, reducing edge-state contributions and consequently the magnitude of the AMR. This is captured in the gate dependence of the edge-state transmission coefficient $\eta$ (Fig. 4b), extracted from our fits to the data of Fig. 4a. The qualitative behaviour of $R_{\mathrm{d}}^{-1}$ is also reasonable, since the dissipative transmission coefficient increases as more electrons are accumulated. Similarly, the temperature dependence of the AMR (Fig. 4c) also captures the competition between the edge states and dissipative channels since our model quantifies edge transport in temperature regimes away from perfect quantization (Fig. 4d). While perfect Hall quantization has required dilution fridge temperatures, our results here demonstrate that the temperature scale for observing this novel AMR, with its origins in the edge states can be significantly higher. Furthermore, both the gate and temperature dependence of the fitting parameters reveal the interplay between the edge states and dissipation channels: an increase in dissipative transmission is accompanied by a reduction in edge transmission and vice versa.

\section{Conclusions}

In summary, we have probed the evolution of edge states in a QAH insulator, as its magnetization is tilted in-plane, both by angular magnetoresistance measurements and by developing a model based on the Landauer-Büttiker formalism that accounts for coexisting dissipative channels. We demonstrate a crossover from dominant 1D edge transport to diffusive transport driven by the closing of the surface state magnetic gap. This crossover results in a novel, gate-tunable, giant AMR effect that serves as a quantitative probe of the interplay between the chiral edge modes and dissipative channels, even in non-ideal QAH regimes. This methodology is relevant to ongoing efforts to raise the temperature scale of the quantum anomalous Hall effect ${ }^{21}$.
Furthermore, as with recent advances that exploit the interplay between spin polarized states in topological insulators and ferromagnetism ${ }^{22-24}$, the observation of a giant AMR effect may provide a useful basis for proof-of-concept devices for 'topological spintronics'.

\section{Methods}

Material synthesis and characterization. $\mathrm{The} \mathrm{Cr}_{x}(\mathrm{Bi}, \mathrm{Sb})_{2-{ }_{-}} \mathrm{Te}_{3}$ thin films studied by transport were grown under ultra-high vacuum (low $10^{-10}$ torr) by molecular beam epitaxy on $\langle 111\rangle$ STO. SQUID magnetometry was however performed on $\mathrm{Cr}$ doped thin films grown on $<111>$ InP substrates. (See Supplementary Fig. 12, Supplementary Note 5). The source material was high purity $\mathrm{Cr}, \mathrm{Bi}, \mathrm{Sb}$ and $\mathrm{Te}$ thermally evaporated from conventional Knudsen cells. For device $\mathrm{C}$ however, e-beam evaporation of $\mathrm{Cr}$ was employed instead. Before growth, the STO substrates were subjected to an ex situ high temperature (925$950{ }^{\circ} \mathrm{C}$ ) anneal in an oxygen atmosphere to obtain a smoother substrate morphology. The topological insulator film of device A was capped in situ with a thin layer of room-temperature-deposited $\mathrm{Al}$, which naturally oxidized upon exposure to atmosphere. Devices B and C were uncapped. The thickness of the topological insulator films was estimated by comparison with the growth rate of calibration samples, whose thickness was measured using high resolution transmission electron microscopy and also confirmed by atomic force microscopy of the Hall bar step edge. The relative compositions of $\mathrm{Cr}, \mathrm{Bi}, \mathrm{Sb}$ and $\mathrm{Te}$ in the different films were obtained by high resolution energy dispersive X-ray spectroscopy and secondary ion mass spectroscopy.

Transport measurements. The thin-film devices were mechanically scratched into Hall bars using a probe station. Since the Hall bars were mechanically defined, our raw data were symmetrized to account for geometric offsets. The angular measurements were performed in an Oxford Triton $\mathrm{He}^{3}$ vector magnet system with a radial field limit of $1 \mathrm{~T}$ and a base temperature of $280 \mathrm{mK}$. All transport measurements typically employed standard low frequency $(19 \mathrm{~Hz})$ lock-in techniques with source currents in the range 10-20 nA, and a Keithley 6430 source meter for back gating.

\section{References}

1. Hasan, M. Z. \& Kane, C. L. Colloquium: topological insulators. Rev. Mod. Phys 82, 3045-3067 (2010).

2. Qi, X.-L. \& Zhang, S.-C. Topological insulators and superconductors. Rev. Mod. Phys. 83, 1057-1110 (2011).

3. Liu, M. et al. Crossover between weak antilocalization and weak localization in a magnetically doped topological insulator. Phys. Rev. Lett. 108, 036805 (2012).

4. Checkelsky, J. G., Ye, J., Onose, Y., Iwasa, Y. \& Tokura, Y. Dirac-fermionmediated ferromagnetism in a topological insulator. Nat. Phys. 8, 729-733 (2012).

5. Zhang, D. et al. Interplay between ferromagnetism, surface states, and quantum corrections in a magnetically doped topological insulator. Phys. Rev. B 86, 205127 (2012).

6. Chang, C.-Z. et al. Experimental observation of the quantum anomalous Hall effect in a magnetic topological insulator. Science 340, 167-170 (2013).

7. Lee, J. S. et al. Ferromagnetism and spin-dependent transport in n-type $\mathrm{Mn}-\mathrm{Bi}_{2} \mathrm{Te}_{3}$ thin films. Phys. Rev. B 89, 174425 (2014).

8. Checkelsky, J. G. et al. Trajectory of the anomalous Hall effect towards the quantized state in a ferromagnetic topological insulator. Nat. Phys. 10, 731-736 (2014).

9. Kou, X. et al. Scale-invariant dissipationless chiral transport in magnetic topological insulators beyond two-dimensional limit. Phys. Rev. Lett. 113, 137201 (2014)

10. Wei, P. et al. Exchange-coupling-induced symmetry breaking in topological insulators. Phys. Rev. Lett. 110, 186807 (2013).

11. Kandala, A. et al. Growth and characterization of hybrid insulating ferromagnet-topological insulator heterostructure devices. Appl. Phys. Lett. 103, 202409 (2013).

12. Lang, M. et al. Proximity induced high-temperature magnetic order in topological insulator-ferrimagnetic insulator heterostructure. Nano Lett. 14, 3459-3465 (2014).

13. Qi, X.-L., Hughes, T. L. \& Zhang, S.-C. Topological field theory of time-reversal invariant insulators. Phys. Rev. B 78, 195424 (2008).

14. $\mathrm{Xu}, \mathrm{S}$.-Y. et al. Hedgehog spin texture and Berry's phase tuning in a magnetic topological insulator. Nat. Phys. 8, 616-622 (2012).

15. Zhang, F., Kane, C. J. \& Mele, E. J. Surface state magnetization and chiral edge states on topological insulators. Phys. Rev. Lett. 110, 046404 (2013).

16. $\mathrm{Yu}, \mathrm{R}$. et al. Quantized anomalous Hall effect in magnetic topological insulators. Science 329, 61-64 (2010). 
17. Thomson, W. On the electro-dynamic qualities of metals:-effects of magnetization on the electric conductivity of Nickel and of Iron. Proc. R. Soc. Lond. 8, 546-550 (1856).

18. McGuire, T. \& Potter, R. Anisotropic magnetoresistance in ferromagnetic 3d alloys. IEEE Trans. Magn. 11, 1018-1038 (1975).

19. Chen, J. et al. Gate-voltage control of chemical potential and weak antilocalization in $\mathrm{Bi}_{2} \mathrm{Se}_{3}$. Phys. Rev. Lett. 105, 176602 (2010).

20. Wang, J. et al. Anomalous edge transport in the quantum anomalous Hall state. Phys. Rev. Lett. 111, 086803 (2013).

21. Chang, C.-Z. et al. High-precision realization of robust quantum anomalous Hall state in a hard ferromagnetic topological insulator. Nat. Mater. 14, 473-477 (2015).

22. Mellnik, A. et al. Spin-transfer torque generated by a topological insulator. Nature 511, 449-451 (2014).

23. Fan, Y. et al. Magnetization switching through giant spin-orbit torque in a magnetically doped topological insulator heterostructure. Nat. Mater. 13, 699-704 (2014).

24. Li, C. H. et al. Electrical detection of charge-current-induced spin polarization due to spin-momentum locking in $\mathrm{Bi}_{2} \mathrm{Se}_{3}$. Nat. Nanotech. 9, 218-224 (2014).

\section{Acknowledgements}

We acknowledge support from DARPA MESO (Grant No. N66001-11-1-4110), ONR (Grant No. N00014-12-1-0117) and ARO MURI (Grant No. W911NF-12-1-0461),

as well as use of the NSF National Nanofabrication Users Network Facility at Penn State. We thank N.P. Ong, Minhao Liu and J. Jain for useful discussions.

\section{Author contributions}

A.K., A.R. and N.S. planned the experiment. A. K and A. R. characterized the films, fabricated the devices and performed transport experiments, A. R. synthesized the samples, S.K. performed SQUID magnetometry measurements. A.K., A.R., C.-X.L. and N.S. analysed the data. A.K., A.R., C.-X.L. and N.S. wrote the manuscript.

\section{Additional information}

Supplementary Information accompanies this paper at http://www.nature.com/ naturecommunications

Competing financial interests: The authors declare no competing financial interests.

Reprints and permission information is available online at http://npg.nature.com/ reprintsandpermissions/

How to cite this article: Kandala, A. et al. Giant anisotropic magnetoresistance in a quantum anomalous Hall insulator. Nat. Commun. 6:7434 doi: 10.1038/ncomms8434 (2015).

(c) (i) This work is licensed under a Creative Commons Attribution 4.0 International License. The images or other third party material in this article are included in the article's Creative Commons license, unless indicated otherwise in the credit line; if the material is not included under the Creative Commons license, users will need to obtain permission from the license holder to reproduce the material. To view a copy of this license, visit http://creativecommons.org/licenses/by/4.0/ 